\title{
PORTACION FARINGEA DE Streptococcus pyogenes Y PERFILES DE SENSIBILIDAD EN ESCOLARES DE CARTAGENA
}

\author{
CARRYING PHARYNGEAL OF Streptococcus pyogenes AND \\ SENSITIVITY PROFILES IN SCHOOLCHILD FROM CARTAGENA
}

\section{TITULO CORTO: PORTACION FARINGEA DE Streptococcus pyogenes}

Lucy Margarita Villafañe-Ferrer ${ }^{1}$ y Raimundo Castro-Orozco ${ }^{2}$

Recibido en febrero 03 de 2015

Aceptado en abril 16 de 2015

\section{RESUMEN}

Determinar la frecuencia de portación faríngea de $S$. pyogenes y sus perfiles de sensibilidad a antibióticos en escolares de la ciudad de Cartagena. Estudio transversal analítico; la muestra estuvo conformada por 131 niños. Las cepas de Streptococcus pyogenes fueron identificadas por métodos convencionales. La sensibilidad a antibióticos fue determinada por el método de Kirby-Bauer. Se aplicó una encuesta para identificar condiciones de riesgo asociados a la portación nasal. De la población estudiada, el 19,8\% fueron portadores de la bacteria. Se obtuvieron 26 aislamientos de Streptococcus pyogenes. Al evaluar la sensibilidad antimicrobiana, se encontró que fueron principalmente sensibles a Ceftriaxona y Eritromicina (84,6\% para cada uno). El 23,1 \% (6/26) de los aislamientos fueron resistentes a ampicilina. No se encontró asociación significativa entre el estado de portación y la presencia de factores de riesgo $(p>0,05)$. Se encontraron cepas resistentes a los antibióticos considerados de primera elección para el tratamiento de infecciones por esta bacteria.

Palabras clave: Streptococcus pyogenes; portador sano; faringe; preescolar; farmacorresistencia microbiana; factores de riesgo. (Fuente: DeCS)

\section{ABSTRACT}

To determine the frequency of carrying pharyngeal of Streptococcus pyogenes and their sensitivity profiles in schoolchildren from Cartagena. Analytical cross-sectional study, the sample was composed by 131 children. Strains of Streptococcus pyogenes were identified using conventional methods. Antibiotic sensitivity was determined the Kirby-Bauer methods. A questionnaire was applied in order to identify risk factors associated.19,8\% of children were carriers of bacterium. 26 isolates of Streptococcus pyogenes were obtained. To evaluate the sensitivity were found strains sensible to ceftriaxone and erytrhomycin (84,6\% each one). $23,1 \%(6 / 26)$ of isolates were resistant

\footnotetext{
1. Magister en Microbiología. Docente investigador. Corporación Universitaria Rafael Núñez. Programa de Bacteriología. Cartagena - Bolívar - Colombia. Correo electrónico: lucy.villafane@curnvirtual.edu.co; villafanelucy@yahoo.com

2. Magister en Microbiología. Doctorando en Medicina Tropical. Universidad de Cartagena. Docente investigador Universidad de San Buenaventura. Cartagena Bolívar - Colombia. Correo electrónico: raimundo_castro_orozco@hotmail.com
} 
to ampicillin. It not was found association between carrying pharyngeal of Streptococcuspyogenes and risk factors ( $p>0,05$ ). It were found resistant strains to antibiotics considered of first election for therapy of infectious diseases produced by this bacterium.

Keywords: Streptococcus pyogenes; carrier state; pharynx; child, preschool; drug resistance, microbial; risk factors. (Fuente: MeSH)

\section{INTRODUCCIÓN}

Streptococcus pyogenes es una bacteria grampositiva, $\mathcal{S}$ causante de infecciones supurativas y no supurativas. Es el patógeno aislado con más frecuencia en casos de faringitis aguda. Además, causa síndromes post-estreptocócicos tales como fiebre reumática y glomerulonefritis ${ }^{1-3}$.

Esta bacteria coloniza con frecuencia la faringe, encontrándose principalmente en niños de escuelas y guarderías, con una tasa de portación del 15 al 20\% ${ }^{1}$. La forma más común de transmisión se realiza de persona a persona a través de gotitas de saliva; de ahí la importancia que presentan los portadores faríngeos y su identificación debido a que son la principal fuente de diseminación. Esta forma de transmisión puede ocasionar brotes epidémicos, principalmente entre contactos familiares o comunidades cerradas como escuelas, internados y guarderías ${ }^{4}$. Esta situación es considerada un problema importante de salud pública, porque la infección frecuentemente conlleva a secuelas postestreptococcicas ${ }^{5}$.

Existen condiciones que favorecen la transmisión de $S$. pyogenes entre los individuos de una comunidad, tales como la edad, hacinamiento, hospitalización previa, uso de antibióticos, condición de fumador pasivo, diabetes, inmunosupresión, estrato socioeconómico bajo, amigdalectomía, condiciones higiénicas y convivir con personas que tienen síntomas de infecciones del tracto respiratorio superior ${ }^{5}$.

Cuando las infecciones por $S$. pyogenes se presentan, el tratamiento que se prescribe son los antibióticos -lactámicos y si el individuo es alérgico se tiene como segunda opción a los macrólidos y lincosamidas. La resistencia de $S$. pyogenes a estos antibióticos ha sido reportada y presenta un comportamiento creciente en el mundo ${ }^{6}$. En diversos países, se han realizado estudios sobre la resistencia a antibióticos en cepas de $S$. pyogenes aisladas de portadores faríngeos y de individuos con los diferentes cuadros clínicos causados por esta bacteria, que proporcionan información acerca de los perfiles de resistencia de las cepas circulantes ${ }^{2,7-10}$. En Colombia, se han realizado estudios para evaluar la portación faríngea en escolares, en las ciudades de Medellín y Bogotát1, ${ }^{12}$; sin embargo, después de utilizar una estrategia de búsqueda bibliográfica (palabras claves: Streptococcus pyogenes, portador) en las principales bases electrónicas de datos (Scielo Colombia, LILACS, PubMed), no se encontraron investigaciones similares realizadas en la ciudad de Cartagena.

Por tal razón el objetivo de este estudio fue determinar la frecuencia de portación faríngea de $S$. pyogenes y sus perfiles de sensibilidad a antibióticos en escolares de la ciudad de Cartagena.

\section{MATERIALES Y MÉTODOS}

Estudio transversal analítico en el que fueron incluidos 131 niños seleccionados por muestreo aleatorio estratificado (error de estimación del 5 \%), a partir de una población total de 199 niños que asistían a una escuela de la ciudad de Cartagena. Los estratos de la muestra correspondieron a los nueve cursos de primaria de la institución educativa. Como criterio de inclusión se estableció la edad de entre 3 a 15 años, y ausencia de signos o síntomas de faringoamigdalitis aguda y que no hayan recibido antibioticoterapia las cuatro semanas previas.

A cada niño se le tomó una muestra de exudado faríngeo mediante un escobillón estéril y se sembró por agotamiento en agar sangre de carnero al $5 \%$ e incubadas por 18 a 24 horas a $37^{\circ} \mathrm{C}$ en ambiente microaerofílico $10 \%$ de $\mathrm{CO}_{2}$.

Para la identificación de S. pyogenes se evaluó: morfología bacteriana, comportamiento tincional, sensibilidad a bacitracina, resistencia a SXT (trimetropin sulfametoxazol), 
pruebas bioquímicas y serológicas (presencia del antígeno de grupo A de Lancefield con la técnica comercial de aglutinación en látex Slidex Strepto-Kit, Biomérieux) ${ }^{12,13}$.

El estudio de la sensibilidad a antibióticos se realizó por el método de difusión con disco mediante la técnica de Kirby-Bauer, siguiendo las recomendaciones y criterios de interpretación establecidos por el Clinical and Laboratory Standards Institute, (CLSI), 2014. Los sensidiscos de antibióticos utilizados fueron Ampicilina (AMP), Ceftriaxona (CRO), Clindamicina (CLI) y Eritromicina (ERY) ${ }^{14,15}$.

A cada aislamiento de $S$. pyogenes se le se realizó el Test $\mathrm{D}$ para la detección de fenotipos de resistencia inducible a clindamicina, siguiendo las recomendaciones del CLSI, $2014^{14,15}$.

Se evaluó la presencia de factores de riesgo asociados a la portación con el diligenciamiento de un cuestionario por parte del padre de familia o representante legal, que previamente firmaron una cláusula de consentimiento informado.

El análisis estadístico de los resultados se realizó con el programa SPSS v.19 para Windows. Para establecer si existía relación entre las variables cualitativas se realizaron tablas de contingencia mediante la prueba $X^{2}$. El nivel de significación estadística considerado fue del $5 \%(\mathrm{p} \leq 0,05)$.

\section{Declaración de aspectos éticos y conflicto de intereses}

Para su realización, esta investigación tuvo en cuenta las normas éticas de la Declaración de Helsinki y la Resolución Nº 8430 de 1993 del Ministerio de Salud y Protección Social de la República de Colombia. Se solicitó a los representantes legales el consentimiento para que los niños pudieran participar en la investigación. Esta investigación fue realizada por profesionales con conocimiento y experiencia para cuidar la integridad del ser humano, con el consentimiento por escrito de los representantes legales de los participantes del estudio. De 114 igual forma se protegió la privacidad de los participantes.

Los autores de este artículo manifiestan que durante la ejecución de la investigación y redacción del manuscrito no han incidido intereses o valores distintos a los que usualmente tiene la investigación.

\section{RESULTADOS}

Se incluyeron 131 niños cuya edad era de $8,3 \pm 1,8$ años (rango: 4 a 13 años), con ligero predominio del sexo femenino $(51,1 \%)$.

En este estudio se encontró que 19,8 \% (26/131; IC95 $\% 13,9$ a 27,5) de los niños fueron portadores de esta bacteria grampositiva y la mayor frecuencia se encontró en el grupo etario de 4-7 años. No se encontró asociación estadísticamente significativa entre la portación de $S$. pyogenes y los grupos etarios $(\mathrm{p}=0,89)$ (Tabla 1$)$.

Tabla 1. Distribución de los portadores según el grupo etario

\begin{tabular}{|c|c|c|c|}
\hline \multirow{2}{*}{$\begin{array}{c}\text { Grupo } \\
\text { etario } \\
\text { (años) }\end{array}$} & \multicolumn{2}{|c|}{ Portación de S. pyogenes } & \multirow{2}{*}{ Total (\%) } \\
\cline { 2 - 3 } & Si & No & \\
\hline $4-7$ & 12 & 43 & $55(42 \%)$ \\
\hline $8-10$ & 11 & 48 & $59(45 \%)$ \\
\hline $11-13$ & 3 & 14 & $17(13 \%)$ \\
\hline Total & 26 & 105 & $\begin{array}{c}131(100 \\
\%)\end{array}$ \\
\hline
\end{tabular}

$X^{2}=0,24 ; p=0,89$

Al evaluar la sensibilidad antimicrobiana de los 26 aislamientos de $S$. pyogenes, se encontró que fueron principalmente sensibles a Ceftriaxona y Eritromicina (84,6\% para cada uno). Entre los aislamientos que fueron resistentes a antibióticos se encontró que el 23,1% (6/26) fueron resistentes a ampicilina (Tabla 2).

Teniendo en cuenta la resistencia de cada cepa a los antibióticos evaluados, se identificaron 10 fenotipos de resistencia (Tabla 3).

$\mathrm{Al}$ aplicar el test $\mathrm{D}$, en el presente estudio, se encontraron dos cepas con fenotipo $\mathrm{CMLS}_{\mathrm{B}}$ y una cepa con fenotipo $\mathrm{iMLS}_{\mathrm{B}}$ (test D positivo) (Tabla 3). 
Tabla 2. Patrones de sensibilidad de los aislamientos de S. pyogenes

\begin{tabular}{|c|c|c|}
\hline Antibióticos & $\begin{array}{c}\text { Sensible } \\
\text { n (\%) }\end{array}$ & $\begin{array}{c}\text { Resistente } \\
\text { n (\%) }\end{array}$ \\
\hline Ampicilina & $20(76,9 \%)$ & $6(23,1 \%)$ \\
\hline Ceftriaxona & $22(84,6 \%)$ & $4(15,4 \%)$ \\
\hline Clindamicina & $21(80,8 \%)$ & $5(19,2 \%)$ \\
\hline Eritromicina & $22(84,6 \%)$ & $4(15,4 \%)$ \\
\hline
\end{tabular}

En cuanto a los factores de riesgo, se encontró que el 19,2 $\%(5 / 26)$ de portadores reportaron ser fumadores pasivos. No se encontró asociación significativa entre el estado de portación y la presencia de este factor de riesgo $(\mathrm{p}=0,71)$.

Tabla 3. Fenotipos de resistencia de Aislamientos de $S$. pyogenes

\begin{tabular}{|c|c|c|}
\hline $\begin{array}{l}\text { Fenotipos de } \\
\text { resistencia }\end{array}$ & Resistente a & Frecuencia \\
\hline FR1 & $\begin{array}{c}\text { AMP, ERY } \\
\text { (Fenotipo M) }\end{array}$ & 1 \\
\hline FR2 & CLI & 1 \\
\hline FR3 & CRO & 2 \\
\hline FR4 & AMP, CLI & 1 \\
\hline FR5 & AMP, CRO & 1 \\
\hline FR6 & $\begin{array}{l}\text { AMP, CLI, } \\
\text { ERY (Fenotipo } \\
\text { cMLS }_{\mathrm{B}} \text { ) }\end{array}$ & 1 \\
\hline FR7 & AMP & 1 \\
\hline FR8 & $\begin{array}{l}\text { ERY (Fenotipo } \\
\text { iMLS }_{\mathrm{B}} \text { ) }\end{array}$ & 1 \\
\hline FR9 & $\begin{array}{l}\text { CLI, ERY } \\
\text { (Fenotipo } \\
\text { cMLS }_{B} \text { ) }\end{array}$ & 1 \\
\hline FR10 & AMP, CLY, CRO & 1 \\
\hline Total & & 11 \\
\hline
\end{tabular}

AMP: ampicilina; CLI: clindamicina; ERY: eritromicina; CRO: ceftriaxona.
En tan solo dos portadores se encontraron antecedentes de catarro de vías aéreas superiores y de convivencia con personas que presentan con frecuencia dolores de garganta.

El resto de factores de riesgo (hospitalización reciente, episodios de faringoamigdalitis por año, amigdalectomía, antecedentes de enfermedad respiratoria alérgica y hacinamiento) no se presentaron en la población estudiada.

\section{DISCUSIÓN}

En Colombia, es relevante realizar este tipo de investigaciones, debido al desconocimiento de la prevalencia de portación y características de cepas de $S$. pyogenes en la población infantil ${ }^{11}$; lo cual es importante para implementar programas efectivos de prevención, como lo establece la OMS en su informe Iniciative for Vaccine Research ${ }^{16}$.

En este estudio se encontró que 19,8 \% (26/131; IC95 $\% 13,9$ a 27,5) de los niños fueron portadores de esta bacteria grampositiva y la mayor frecuencia se encontró en el grupo etario de 4-7 años; este porcentaje es similar a lo reportado en escolares de la Habana, $\mathrm{Cuba}^{17}$, y en niños de 3 a 13 años de dos ciudades colombianas ${ }^{11}$. Sin embargo, es superior a la encontrada por otros autores en poblaciones similares ${ }^{18-20}$. Estos resultados son coherentes con la literatura en la que se menciona que en la población infantil, la tasa de portación de esta bacteria varía de 15 a $20 \%$; siendo más frecuente en niños entre 5-15 años ${ }^{2,11,21}$.

Los resultados de esta investigación, difieren de los encontrados por otros autores los cuales reportan que todas las cepas de $S$. pyogenes aisladas fueron sensibles a betalactamicos ${ }^{6,10,12,22}$. En cuanto a la resistencia a macrólidos y lincosamidas, los porcentajes reportados en este estudio son mayores a los encontrados en otras investigaciones ${ }^{1,12,18,23}$. La resistencia a estos antibióticos considerados de primera elección, tiene implicaciones en el pronóstico de infecciones por estas cepas ${ }^{6,24}$.

$\mathrm{Al}$ aplicar el test $\mathrm{D}$ en el presente estudio, se encontraron dos cepas con fenotipo $\mathrm{cMLS}_{\mathrm{B}}$, una cepa con fenotipo $\mathrm{iMLS}_{\mathrm{B}}$ (test D positivo) y otra cepa con fenotipo M. (Tabla 2). 
Estos fenotipos han sido reportados previamente ${ }^{6,7,25}$. El fenotipo $\mathrm{CMLS}_{\mathrm{B}}$, expresa resistencia constitutiva a la eritromicina y la clindamicina, la cual está dada por una metilasa del ARN ribosomal 23S codificada por el gen ermB, cuya acción bloquea la unión del antibiótico y el fenotipo iMLS $S_{B}$, de resistencia constitutiva a macrólidos e inducible a lincosamidas, es determinado por medio de una metilasa ErmA codificada por el gen ermA caracterizada por un achatamiento en el halo de la clindamicina en la proximidad de la eritromicina (D- test positivo) ${ }^{26-28}$.

Como se mencionó anteriormente, a partir de una búsqueda bibliográfica no se encontraron investigaciones similares en la ciudad de Cartagena. En cambio, en Colombia existe un referente ${ }^{12}$ publicado en el año 2009, en el que se reportan porcentajes de resistencia a macrólidos y lincosamidas inferiores a los de este estudio; sin embargo, no reportan cepas resistentes a ampicilina.

En cuanto a los factores de riesgo, Fuentes et al. ${ }^{17}$ reportaron diferencias estadísticas significativas entre éstos y el estado de portación en una población infantil de la Habana, Cuba; de igual forma, White et al. ${ }^{29}$, reportaron diferencias significativas, pero la población estudiada fue diferente.

Estos factores de riesgo pueden potencializar o aumentar la susceptibilidad de un individuo para ser portador o desarrollar un cuadro clínico por esta bacteria ${ }^{22,29,30}$, sin embargo, la presencia de estos factores de riesgo no fue representativa en los escolares analizados.

Los resultados encontrados en este estudio servirán de base para el diseño y aplicación de estrategias de prevención.

\section{LIMITACIONES DEL ESTUDIO}

Esta investigación tiene limitaciones relacionadas con la validez externa por la imposibilidad de generalizar los resultados obtenidos en la población estudiada. No se utilizaron técnicas de biología molecular lo cual hubiera permitido la confirmación de los aislamientos y

116 la determinación de clones circulantes $(8,19)$.

\section{DECLARACIÓN SOBRE CONFLICTO DE INTERESES:}

Los autores de este artículo manifiestan que durante la ejecución de la investigación y redacción del manuscrito no han incidido intereses o valores distintos a los que usualmente tiene la investigación.

La presente investigación fue financiada con recursos propios.

\section{REFERENCIAS BIBLIOGRÁFICAS}

1. Dumre S, Sapkota K, AdhikariN, Acharya D, Karki $\mathrm{M}$, Bista $\mathrm{S}$, et al. Asymptomatic throat carriage rate and antimicrobial resistance pattern of Streptococcus pyogenes in Nepalese school children. Kathmandu Univ Med. 2009; 7(28):392-6.

2. Rijal K, Dhakal N, Shah R, Timilsina S, Mahato P, Thapa et al. Antibiotic susceptibility of Group A Streptococcus isolated from throat swab culture of school children in Pokhara, Nepal. Nepal Med Coll J. 2009; 11(4): 238-40.

3. Nunes M, SáFigueiredo A, Ferreira-Carvalho B. Antimicrobial susceptibility patterns and genomic diversity in strains of Streptococcus pyogenes isolated in 1978-1997 in different Brazilian cities. J Med Microbiol. 2003; 52(Pt 3): 251-8.

4. Gutiérrez C, Sibrian B, Chacón M, Pérez-Ybarra L, Caceras $\mathrm{J}$, Valdes $\mathrm{N}$, et al. Streptococcus betahemolíticos en faringe de estudiantes, municipio Francisco Linares Alcántara, Estado Aragua. Odous Científica. 2012; 13(2): 15 - 22.

5. Durmaz R, Durmaz B, Bayraktar M, Halil I, Tayyar M, Aktas E, et al. Prevalence of group A streptococcal carriers in asymptomatic children and clonal relatedness among isolates in Malatya, Turkey. J. Clin. Microbiol. 2003; 41(11): 5285-7.

6. Rodriguez C, Rojas P, Wozniak A, Kalergis A, Ceron I, Riedel I, et al. Análisis de los fenotipos y genotipos de resistencia a eritromicina y clindamicina en cepas de Streptococcus pyogenes aisladas en Chile en un período de 10 años. Rev Med Chil. 2011; 139(9): 1143-9.

7. Rubio V, Valdezate S, Álvarez D, Medina M, Salcedo C, Saez- Nieto J. Molecular epidemiology, antimicrobial susceptibilities and resistance mechanisms of Streptococcus pyogenes isolates resistant to erythromycin and tetracycline in Spain (1994-2006). BMC Microbiol. 2012; 12:215- 26.

8. Pires R, Rolo D, Morais A, Brito-Avo A, Johansson C, Henriques-Normark B, et al. Description of macrolideresistant and potential virulent clones of Streptococcus pyogenes causing asymptomatic colonization during 2000-2006 in the Lisbon area. Eur J Clin Microbiol Infect Dis. 2012; 31(5):849-57.

9. Ksia S, Smaoui H, Kechrid A, Bouvet A. Streptococcus pyogenes isolated in a Tunisian pediatric population: Emm types, $T$ types, virulence factors and genes of resistance to macrolide and tetracycline. Malays $\mathrm{J}$ Microbiol. 2013; 9(1):24-32. 
10. Malik U, Saba N, Qazilbash A. Antibiogram sensitivity Pattern of Streptococcus pyogenes and Streptococcus pneumoniae isolated from patients with sore throat and pneumonia infections. Pak J Biol Sci. 2005; 8(8):1145-51.

11. Restrepo MA, Múnera MI, Ramírez S, Acuña C. Infección y colonización faríngea asintomática de niños por Streptococcus pyogenes. Iatreia. 2012; 25(3): 203-9.

12. Giraldo R. Susceptibilidad del estreptococo beta hemolítico del grupo A. Revista medicina. 2009; 8(1):26-9.

13. Harley J, Prescott L. Exercise 56: Streptococci. In: Harley J, Prescott L. Harley- Prescott: Laboratory Exercises in Microbiology, Fifth Edition. McGraw-Hill Company. 2002. p. 347-51.

14. Giannelli S, Posse G. Prevalencia de portación asintomática del estreptococo $\beta$ - hemolítico grupo A (Streptococcus pyogenes). Arch Argen Pediatr. 2007; 105(3): 221-4.

15. Clinical and Laboratory Standards Institute. Performance standards for antimicrobial susceptibility testing: twentyfourth informational supplement. CLSI document M100-S24. Wayne, PA: CLSI; 2014.

16. World Health Organization. Iniciative for Vaccine Research (IVR) [Internet].[citado 7 enero 2015] Disponible en: http://www.who.int/vaccine_research/ diseases/soa_bacteriaI/en/index.

17. Fuentes Y, Martínez I, Sierra G, Pérez L, López O, Valdés M. Colonización faríngea por bacterias potencialmente patógenas en niños sanos de una escuela primaria. Rev Cubana Med Trop.2009; 61(1):50-6.

18. Maciel A, Da Silva I, De Souza A, Malagueño E, Sekiguchi T, De Andrade P. Portadores assintomáticos de infecções por Streptococcus pyogenes emduasescolas públicas na cidade do Recife, Pernambuco. Rev Bras Saúde Matern Infant., Recife. 2003; 3(2): 175-80.

19. Perçin D, Bozdoğan B, Ayangil D, Sümerkan B, Appelbaum P. Molecular Epidemiology and Antibacterial Susceptibility of Streptococci Isolated from Healthy Children Attending Day Care Units. Balkan Med J. 2011, 28: 414-9.
20. Shaikh N, Leonard E, Martin J. Prevalence of Streptococcal Pharyngitis and Streptococcal Carriage in Children: A meta-analysis. Pediatrics. 2010; 126(3): 556- 64.

21. García M. Comportamiento de los estreptococos betahemolíticos en escolares. Sanidad militar. 2012; 68(1): 17-21.

22. Garcia G, Mosquera C. Faringo- Amigdalitis. En: Ucros S, Mejía N. Guías de pediatría práctica basadas en la evidencia. Primera edición. Bogotá: Editorial Médica Internacional; 2009.

23. Cockerill FR, MacDonald KL, Thompson RL, Roberson F, Kohner PC, Besser-Wiek J, et al. An outbreak of invasive group A streptococcal disease associated with high carriage rates of the invasive clone among schoolaged children. JAMA.1997; 277(1):38-43.

24. Casella JM. Resistencia a los antibacterianos en América Latina: consecuencias para la infectología. Rev Soc Bol Ped. 2012; 51(2): 109 - 24.

25. Fariña N, Ocampos M, Laspina F, Balmaceda M, Sanabria R, Samudio M. Estreptococo beta hemolítico grupo A. Resistencia a los macrólidos. Mem. Inst. Investig. Cienc. Salud. 2001; 1(1): 72-5.

26. Jaiswal S, Pandey R, Sharma B. Reduction of antibiotic resistance in bacteria: a review. International Journal Pharmaceutical Sciences and Research. 2012;3(1): 695-9.

27. Gonzales A, Ortiz C, Mota R, Dickinson E, Dávila R, Fernández M. Sensibilidad antimicrobiana y caracterización de cepas de Streptococcus pyogenes aisladas de un brote de escarlatina. Salud Pública de México. 2002; 44 (5):437- 41.

28. Espinosa B, Martínez M, Sanchez M, Wertheimer A. The determinants of the antibiotic resistance process. Infect Drug Resist. 2009; 2:1-11.

29. White L, Martínez I, Fuentes Y, Valdés M, Izquierdo L. Colonización de bacterias potencialmente patógenas en la faringe de adultos sanos y factores de riesgo asociados. Panorama Cuba y Salud. 2011;7(1):24-30.

30. Braito A, Galgani I, Mohammed R, Iozzi C, Ame M, Haji $\mathrm{H}$ et al. Epidemiology of streptococcus group A in school aged children in Pemba. East African Med J. 2004; 81 (6): 307-12.

Para citar este artículo: Villafañe-Ferrer L, Castro-Orozco R. Portación faríngea de Streptococcus pyogenes y perfiles de sensibilidad en escolares de Cartagena. Duazary. 2015 dic; 12 (2): 112 - 117 